\title{
The evolution of striped coat patterns in African mammals-what function do the stripes have?
}

\author{
Gabriela Vaduva \\ Department of Biology, Lund University, Lund 22362, Sweden \\ E-mail: vaduva_gabriela@yahoo.se
}

Received: November 24, 2015 Accepted: December 20, 2015

doi:10.5296/jbls.v7i1.8613 URL: http://dx.doi.org/10.5296/jbls.v7i1.8613

\begin{abstract}
Several families of mammals have stripes and many hypotheses have been proposed to explain the evolution of the striped coat patterns. However, there is very limited experimental evidence to support which hypotheses are the most likely to explain the evolution of the striped patterns. Therefore, what adaptive function the stripes serve in all these species is still largely unknown. In this study I investigated the characteristics of stripes such as stripe width for both white and black stripes on 36 striped African mammals' coats collected in four museums and three countries. My study further revealed that the appearance of stripes on mammalian coats is most likely not involved in sexual selection. There were close similarities in stripes number or stripes width between sexes for Equus grevyi and Tragelaphus imberbis. In order to evaluate the effects of stripes on tabanids attractiveness, two experiments designed to test tabanids attraction were performed. Data on tabanids daily activity revealed that the flies were more active on partly cloudy and sunny weather. Especially, Haematopota pluvialis is known to have a high flight intensity in cloudy weather before an anticipated rain. Using sticky panels and models, tabanids were more attracted to sunny sides in the both experiments. Moreover, the findings revealed that the stripes in all striped patterns were extremely important in stimulating tabanid flies' repellency. My results suggest that the appearance of stripes on different mammalian coats could be an evolutionary force reducing the attraction of tabanids to many striped animals, consequently, reducing disease transmission risk.
\end{abstract}

Keywords: Zebra, African mammals, Stripes, Tabanidae, Attractiveness, Panels, Models

\section{Introduction}

The behavior of animals and their physical environment are selective factors that drive the evolution of animal color patterns. Color patterns in animals are adaptive and may be used for three reasons: communication, camouflage and physico-physiological functions (Ortolani, 
1999). Animal color patterns are genetically determined. All three explanations are generally accepted, but the mechanism by which the patterns evolved is unknown (Murray, 1981). During the centuries many zoologists tried to explain the function of colors in animals, especially, the significance of black and white coats. Patches of black and white fur create striking contrast in comparison with other color combinations. Color patterns could be aposematic and cryptic in terms of the distance from which they are viewed. Some species that live in dark shadow or white snow show pattern blending. Furthermore, disruptive coloration is, characterized through contrasting colors (Caro, 2009). Dark spots, and horizontally and vertically striped coats have been suggested to have evolved for camouflage which has found some support in comparative analyses (Ortolani, 1999). To understand what function the stripes serve in many African mammals, especially in zebras, it is important to review the many ideas that have tried to explain the pattern and to ask whether the stripes could act as protection to avoid attacks by tabanids flies. One of the most interesting striped patterns is found in the zebra and it continues to be a controversial subject for research and scientific debate. Why did the zebra evolve its stripes? The pattern is formed by roughly horizontal stripes on the legs, rump, and back and relatively vertical stripes on the abdomen, neck and head. The width of the white and black/dark stripes varies in all individuals of the different species of zebras (Kingdon, 1979).

There are three extant species of zebra belonging to the order Perissodactyla, family Equidae: Common zebra, Equus quagga, formerly Equus burchellii (with four subspecies, Equus quagga burchellii, Equus quagga boehmi, Equus quagga crawshayi and the extinct, Cape Quagga, Equus quagga quagga). These are distributed within a vast range across eastern and southern Africa. The Mountain Zebra, Equus zebra (with two subspecies) inhabits south-western Angola, Namibia and also South Africa, and Grévy's Zebra, Equus grevyi, lives in northern Kenya with outliers in Ethiopia and, perhaps, in Somalia (Kingdon, 1979; Bard, 1981). Several explanations for the striped coat pattern in zebras have been proposed, including social benefits, predator avoidance, thermoregulation and protection against tsetse flies, Glossina species (Ruxton, 2002). In terms of social benefit there are some opinions that may be considered. In each species of zebra, the horizontal leg stripes and vertical stripes on the trunk are differently arranged. The pattern of the coat of zebras has been compared after conclusive evidence with human finger prints and in a group the members may identify one another especially by sight using the coat patterns. The stripes may have evolved as foci for grooming behavior and a better use of this mechanism would require an overall covering of the body with stripes. In wild stallions of Equus grevyi, the connection between physical grooming and keeping of close bonds among individuals has been confirmed. Mutual grooming on the back, shoulder and neck is characteristic of mares and their foals. Another function of stripes, in some circumstances, might be to space zebras. Among Equus grevyi individuals, spacing is influenced by the narrower frequency of white and black stripes. In Equus quagga, spacing is determined during the third week, in Equus zebra the fourth week and in Equus grevyi the fifth week (Kingdon, 1979; Murray 1981). Regarding predator avoidance, the stripes are thought to create an optical illusion which makes the apparent size of the zebras larger, thereby confusing predators related to the distance when jump off for the animal. The stripes also create a dazzling effect when the zebra is in close proximity (Morris, 
1991).

Striped pattern may provide camouflage through obliterated shadowing by means of pattern mixing (Ortolani, 1999), which could act as a protection in tall grass. Kingdon (1979) and Morris (1991) argue against this because zebras graze in open areas and never attempt to hide in tall grasses of the savannah. The stripes may in this situation play a role in camouflage only at dusk when the resolving power of the eyes decrease, the stripes color blends toward gray and zebras become cryptic (Godfrey, et al., 1987).

With respect to thermoregulation the stripes may act as a mechanism by which rotary breezes can be produced by differential cooling of the white and black bands (Morris, 1991), resulting in an overall cooling of the animal's skin.

With regards to protection from tsetse flies, the striped coat pattern of zebras has been proposed as a means to prevent their attacks. As such, the stripes might disrupt the visual system of biting tsetse flies (Ortolani, 1999; Ruxton, 2002). All these hypotheses have been discussed and commented upon by Ruxton (2002) and Caro (2009) and both authors consider the hypotheses to be experimentally unconfirmed. This opinion suggests that the function of zebra stripes continues to be unsolved.

Striped patterns in mammals are also found in many other animals belonging to the order Cetartiodactyla and Carnivora apart from zebras.

In the family Giraffidae, Okapi, Okapia johnstoni, that inhabits the Semliki Valley, has stripes on its hindquarters and legs that play a role in communication to conspecifics and also between mother and calf when the calf is following her or sucking. The stripes may also serve in courtship between male and female and the purpose relies on reproductive function (Kingdon, 1979).

The family Bovidae contains many species that possess the visual effect and illusion of stripes on their coat patterns. These stripes have been proposed to be important for communication between the young and its mother (Kingdon, 1982).

Carnivores also display a variety of color patterns (spots, stripes, blotch and so on) that are thought to provide camouflage, have physico-physiological functions or be used in communication (Ortolani, 1999).

Protection from tsetse flies is the only experimentally tested theory for the evolution of striped coat patterns, but the result is still not conclusive (Ruxton, 2002). On the basis thereof, a new possible explanation may be that the striped pattern can be an evolutionary trick to reduce the animal's attractiveness to tabanids flies, in order to avoid attacks by the bloodsucking flies (Horváth, et al., 2008).

Horse flies and deer flies (Diptera: Tabanidae) have a more or less worldwide distribution being absent only at extreme southern and northern latitudes. Adult tabanids flies live in terrestrial habitats, but they lay their eggs on vegetation close to fresh water surfaces. Their larvae develop in mud or water. The source of food for adult tabanids flies is pollen and nectar, but in addition at the onset of eggs development the females feed on blood from 
animals including humans. This makes them vectors of diseases and parasites such as Loa loa, anthrax, Lyme disease, cholera and tularemia. The attacks of tabanids flies also have an important negative effect by reducing milk production and causing weight loss in cattle and horses (Foil, 1989; Lehane, 2005; Horváth, et al., 2008).

It was recently discovered that the polarization vision of tabanids flies is important for explaining their attraction to artificial or natural sources of horizontally polarized light. It has been experimentally demonstrated that the color of the host plays an important role in tabanids attractiveness and that they are more attracted by dark than white horses (Horváth, et al., 2010).

In the present study I have investigated the characteristics of striped coat patterns and in which families of African mammals the striped patterns have evolved. I also measured the stripe width of both white and black stripes on 36 striped coat patterns of African mammals. In addition to the museum measurements I have studied the effect of stripes on the attractiveness of tabanids flies by performing attraction experiments in the field by imitating naturally striped coat patterns. My hypothesis was that the introduction of a striped coat pattern would decrease the attractiveness to tabanids flies. I also investigated whether striped patterns may be involved in sexual selection by comparing stripe patterns between sexes for Equus grevyi and Tragelaphus imberbis.

\section{Material and Methods}

\subsection{Measurements of Stripe Width in Zebra and Some African Mammal Coats}

I took measurements of the characteristics of striped patterns (black/dark and white/light) on museum coats and stuffed animals of African mammals. Measurements of animals were taken from the following locations: Zoological Museum, Lund (on 11, 17, 18/06/2010), Hunting Museum “August von Spiess”, Romania (on 22/07/2010), The Swedish Museum of Natural History, Stockholm (on 16/11/2010) and Natural History Museum of Denmark, Copenhagen (on 17/02/2011).

I measured the stripe width on Equus quagga boehmi (2 coats); Equus grevyi (1 coat and 2 stuffed); Equus burchellii (1 coat and 1 stuffed); Equus quagga burchellii (1 stuffed); Equus zebra hartmannae (1 coat); Tragelaphus eurycerus isaaci (1 stuffed); Tragelaphus strepsiceros (1 coat and 2 stuffed); Tragelaphus imberbis (1 coat and 3 stuffed); Tragelaphus scriptus (2 stuffed); Okapia johnstoni (1 stuffed); Hyaena hyaena (1 coat and 1 stuffed); Civettictis civetta (3 coats and 1 stuffed); Proteles cristatus (1 coat and 2 stuffed); Ictonyx striatus (1 coat and 2 stuffed); Mungos mungo (2 stuffed); Galidictis fasciata (1 stuffed) and Poecilogale albinucha (1 stuffed), using a digital slide-gauge and measuring the stripe width to the nearest $\mathrm{mm}$. The measurements of stripes width on zebra coats were taken only on the right side on approximately uniform regions with stripes such as head (front), side of neck, abdomen, rump, back (if existing), front leg and hind leg. With regards to other African mammals I took measurements in the same way and on the same side (right) where stripes existed.

I used statistical methods to represent the range of mean stripe width (minimum of Mean - 
SD and maximum of Mean + SD) of white and black stripes on different body parts for each stripe measured and also on related species. Descriptive statistics were performed for each dataset. These measurements were used in order to point out that each individual is unique in its striped pattern. If stripes perhaps evolved as a signal specific for each sex and thu s used in sexual selection I tested if the pattern was different between sexes for the species where male and female individuals were found, i.e. Equus grevyi and Tragelaphus imberbis. I expected males to carry a more spectacular, colorful and striped coat, if sexual selection is based on female mate choice.

\subsection{Tabanids Attraction Experiments}

The two experiments were performed at a cattle farm in Vankiva $\left(56^{\circ} 12^{\prime} \mathrm{N}, 13^{\circ} 45^{\prime} \mathrm{E}\right)$ close to Hässleholm, Sweden between 30 June and 7 August 2011. In the first experiment, twenty glossy plastic boards $(50 \times 50 \mathrm{~cm})$ were prepared and divided into four groups (Figure 1). Three groups were placed vertically at $10 \mathrm{~cm}$ above the ground and one group was placed horizontally on the ground. All four groups were placed in a straight line. Each group contained two control surfaces (black and white), a zebra pattern and two Tragelaphidae striped patterns (one with black wide and thin white stripes and the other one having white wide and black thin stripes). Each group had a different orientation of the stripes (vertical, oblique and horizontal). The black and white stripes of the zebra pattern were equal in width $(2.5 \mathrm{~cm})$ whereas for Tragelaphidae I used two configurations: $6 \mathrm{~cm}$ black and $1 \mathrm{~cm}$ white, as well as $6 \mathrm{~cm}$ white and $1 \mathrm{~cm}$ black. These widths were close to my average measurements on the museum skins of these animals. In the second experiment I built four animal models (Figure 2): zebra (vertical, oblique and horizontal stripes), Tragelaphidae (vertical stripes), Okapi (black body surface and horizontal stripes), Hyaenidae (vertical and horizontal stripes) and two control surfaces (black and white). In all models I used white glossy oilcloth painted with glossy black paint. This material was mounted on wood frames $(90 \times 60 \mathrm{~cm})$ placed at the same height as the panels $(10 \mathrm{~cm})$ above the ground. In each animal, the area corresponding to abdomen measured $60 \times 60 \mathrm{~cm}$ whereas the other areas (back and legs) corresponded to an area of $30 \times 60 \mathrm{~cm}$. For the zebra model the $30 \times 60 \mathrm{~cm}$ area was divided in two equal surfaces $(30 \times 30 \mathrm{~cm})$ corresponding to oblique and horizontal striped parts of the body. The black and white control surfaces were $90 \times 60 \mathrm{~cm}$. In all animals the stripe widths represented the average for black and white stripes of each part of body from all individuals and species within the same group (depending on stripe orientation) after my measurements on animal skins in the museums.

For both experiments, panels and models, the orientation was N-S with a sunny (S) and shady $(\mathrm{N})$ side. Both sides were covered with colorless, odorless and transparent insect monitoring glue. The glue was refreshed twice a week. The order of panels in each group were changed randomly once per week. The panels were installed at $50 \mathrm{~cm}$ from each other and the distance between groups was $1 \mathrm{~m}$. With regards to models, the distance between them was ca $5 \mathrm{~m}$ and the angle between the two sides was ca $31^{\circ}$. One of the two surfaces of the panels and models were directed S (sunny side), while the other side was directed N (shady side). 


\section{I Macrothink}
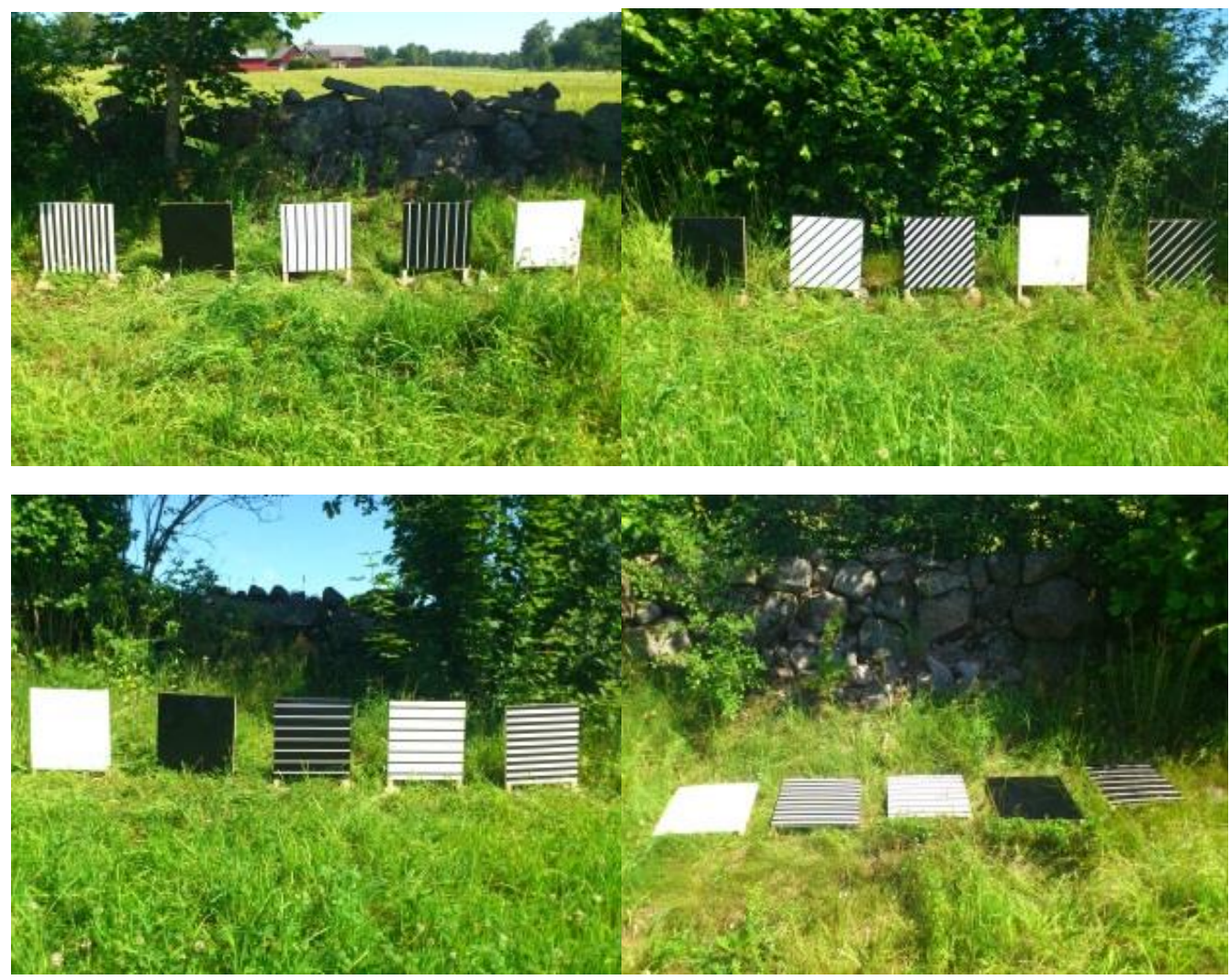

Figure 1. First experiment with four groups of glossy sticky panels. Each group of panels displayed a different stripe orientation (vertical, oblique and horizontal) and two control surfaces (black and white).

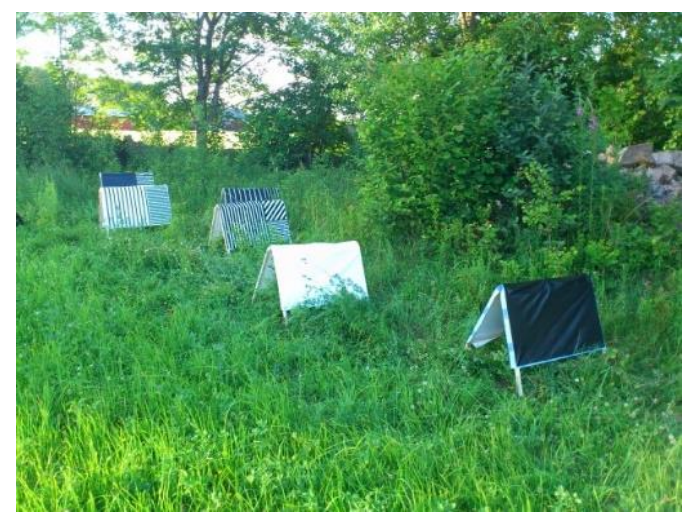

Figure 2. Second experiment with glossy sticky models. Okapi, Hyaenidae, Tragelaphidae and Zebra models and two control surfaces (black and white).

The tabanids flies were collected daily at $19.00 \mathrm{~h}$ in the evening and preserved in $70 \%$ ethyl alcohol.

\subsection{Tabanids Identification}

Three insect field guides (Chvála, et al., 1972; Mandahl-Barth and Coulianos 1985; Chinery, 2007) complemented by a key were used to distinguish flies. Taxonomic identification of 


\section{Ml Macrothink}

Journal of Biology and Life Science

ISSN 2157-6076

2016, Vol. 7, No. 1

specimens trapped on both panels and animal models was performed at the Zoological Museum in Lund by Mikael Sörensson and myself. Five tabanid species were identified (Haematopota pluvialis $90.05 \%$ (out of the total number), Heptatoma pellucens $3.59 \%$, Tabanus bovinus $3.01 \%$, Chrysops viduatus $2.66 \%$ and Hybomitra spp. $0.69 \%)$.

Statistical analyses were performed using SPSS 17.0.

\section{Results}

\subsection{Measurements of Stripe Width in Zebra and Some African Mammal Coats}

Descriptive statistics represent the mean of white and black stripe width variation function of body parts. They showed different values for individuals in each species (Table 1). When comparing male and female of Equus grevyi in terms of the number of stripes, (in order to test if stripes are possibly involved in sexual selection and thus distinct for the sexes), the number of stripes across the body surface was very similar ( $n=242$ for male and $n=243$ for female) as was mean stripes width. In the case of Tragelaphus imberbis the result revealed the same number of stripes across the body surface between a female and one of the males $(n=20)$ and was very similar between the same female and the other males $(n=20$ for female and $n=16$ for each male). Likewise, mean stripes width also showed close similarity between males and female (Table 1).

Table 1. Width w (Mean \pm SD in $\mathrm{cm}$ ) of white and black stripes on different body parts for all measured species.

\begin{tabular}{|c|c|c|c|c|c|c|c|c|}
\hline \multirow{3}{*}{ Species } & \multirow{3}{*}{ 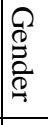 } & & & & & & & \\
\hline & & \multicolumn{2}{|c|}{ Head } & \multicolumn{2}{|c|}{ Side of neck } & \multicolumn{2}{|c|}{ Abdomen } & \multirow{2}{*}{$\begin{array}{l}\text { Rump } \\
\text { white }\end{array}$} \\
\hline & & white & black & white & black & white & black & \\
\hline \multirow[t]{2}{*}{ Equus quagga boehmi } & - & $0.40 \pm 0.12$ & $0.69 \pm 0.26$ & $1.30 \pm 0.57$ & $2.79 \pm 1.87$ & $2.99 \pm 0.88$ & $3.79 \pm 0.99$ & $3.06 \pm 0.09$ \\
\hline & M & $0.58 \pm 0.17$ & $0.89 \pm 0.27$ & $1.96 \pm 0.27$ & $3.94 \pm 0.66$ & $3.75 \pm 0.96$ & $4.12 \pm 1.24$ & $3.42 \pm 2.02$ \\
\hline \multirow{3}{*}{ Equus grevyi } & M & $0.45 \pm 0.20$ & $1.00 \pm 0.35$ & $2.10 \pm 0.76$ & $3.98 \pm 1.66$ & $1.66 \pm 0.20$ & $3.18 \pm 0.37$ & $1.94 \pm 0.20$ \\
\hline & M & $0.48 \pm 0.12$ & $0.52 \pm 0.12$ & $1.78 \pm 0.79$ & $3.27 \pm 1.31$ & $1.60 \pm 0.32$ & $3.16 \pm 0.66$ & $1.55 \pm 0.61$ \\
\hline & $\mathrm{F}$ & $0.45 \pm 0.14$ & $0.59 \pm 0.20$ & $1.77 \pm 0.55$ & $3.74 \pm 1.98$ & $1.35 \pm 0.28$ & $2.66 \pm 0.74$ & $1.78 \pm 0.39$ \\
\hline \multirow[t]{2}{*}{ Equus burchellii } & $\mathrm{F}$ & $0.52 \pm 0.21$ & $0.51 \pm 0.28$ & $2.66 \pm 0.86$ & $3.20 \pm 1.06$ & $5.03 \pm 0.65$ & $3.76 \pm 1.40$ & $4.68 \pm 0.69$ \\
\hline & - & $0.73 \pm 0.30$ & $1.00 \pm 0.30$ & $2.35 \pm 1.12$ & $3.51 \pm 1.86$ & $4.78 \pm 1.06$ & $6.18 \pm 1.22$ & $5.24 \pm 1.13$ \\
\hline Equus quagga burchellii & $\mathrm{M}$ & $0.41 \pm 0.18$ & $0.31 \pm 0.18$ & $1.85 \pm 0.65$ & $3.24 \pm 1.44$ & $3.76 \pm 1.94$ & $3.06 \pm 1.30$ & $5.95 \pm 3.80$ \\
\hline Equus zebra hartmannae & - & $0.50 \pm 0.16$ & $0.49 \pm 0.23$ & $2.19 \pm 0.63$ & $3.91 \pm 1.61$ & $3.02 \pm 0.50$ & $4.40 \pm 0.47$ & $3.88 \pm 0.42$ \\
\hline Tragelaphus eurycerus isaaci & $\mathrm{M}$ & - & - & - & - & $1.60 \pm 1.03$ & $4.85 \pm 1.29$ & - \\
\hline \multirow{3}{*}{ Tragelaphus strepsiceros } & $\mathrm{M}$ & - & - & - & - & $1.09 \pm 0.18$ & $7.83 \pm 6.10$ & - \\
\hline & $\mathrm{M}$ & - & - & - & - & $1.03 \pm 0.15$ & $15.84 \pm 4.62$ & - \\
\hline & $\mathrm{M}$ & - & - & - & - & $0.81 \pm 0.09$ & $14.37 \pm 9.56$ & - \\
\hline \multirow[t]{4}{*}{ Tragelaphus imberbis } & $\mathrm{M}$ & - & - & - & - & $1.07 \pm 0.29$ & $6.86 \pm 2.09$ & - \\
\hline & $\mathrm{M}$ & - & - & - & - & $1.24 \pm 0.13$ & $7.91 \pm 3.27$ & - \\
\hline & $\mathrm{M}$ & & - & - & - & $1.17 \pm 0.24$ & $4.73 \pm 1.04$ & - \\
\hline & $\mathrm{F}$ & - & - & - & - & $0.91 \pm 0.19$ & $5.54 \pm 1.52$ & - \\
\hline \multirow[t]{2}{*}{ Tragelaphus scriptus } & $\mathrm{M}$ & - & - & - & - & $1.56 \pm 0.31$ & $10.02 \pm 0.06$ & - \\
\hline & $\mathrm{F}^{*}$ & - & - & - & - & $0.62 \pm 0.05$ & $2.01 \pm 0.88$ & - \\
\hline Okapia johnstoni & $M$ & - & - & - & - & - & - & $1.50 \pm 0.72$ \\
\hline
\end{tabular}




\begin{tabular}{|l|c|c|c|c|c|c|c|c|}
\hline Hyaena hyaena & $\mathrm{M}$ & - & - & - & - & $3.28 \pm 1.74$ & $2.37 \pm 0.91$ & - \\
& - & - & - & - & - & $3.01 \pm 0.83$ & $2.51 \pm 0.99$ & - \\
\hline \multirow{4}{*}{ Proteles cristatus } & - & - & - & - & - & $3.84 \pm 1.35$ & $1.03 \pm 0.21$ & - \\
\hline & - & - & - & - & - & $4.37 \pm 0.94$ & $1.90 \pm 0.35$ & $2.60 \pm 1.52$ \\
\cline { 2 - 9 } & - & - & - & - & - & $3.79 \pm 1.99$ & $1.23 \pm 0.39$ & - \\
\hline \multirow{5}{*}{ Civettictis civetta } & - & - & - & - & $1.21 \pm 1.12$ & - & - & - \\
\cline { 2 - 10 } & - & - & - & $1.72 \pm 0.64$ & $5.08 \pm 3.98$ & - & - & - \\
\hline \multirow{3}{*}{ Ictonyx striatus } & - & - & - & $1.17 \pm 0.18$ & $2.09 \pm 1.75$ & - & - & - \\
\cline { 2 - 11 } & $\mathrm{F}$ & - & - & $1.85 \pm 0.60$ & $1.97 \pm 1.64$ & - & - & - \\
\hline Mungos mungo & - & - & - & - & - & - & - & - \\
& $\mathrm{M}$ & - & - & - & - & - & - & - \\
\hline Galidictisfasciata & - & - & - & - & - & - & - & - \\
\hline Poecilogale albinucha & $\mathrm{M}$ & - & - & - & - & - & - & - \\
\hline
\end{tabular}

Table 1 continued

\begin{tabular}{|c|c|c|c|c|c|c|c|c|}
\hline \multirow{3}{*}{ Species } & \multirow{3}{*}{ 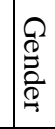 } & \multirow{3}{*}{$\begin{array}{l}\text { Rump } \\
\text { black }\end{array}$} & \multirow{2}{*}{\multicolumn{2}{|c|}{ Back }} & \multirow{2}{*}{\multicolumn{2}{|c|}{ Front leg }} & \multirow{2}{*}{\multicolumn{2}{|c|}{ Hind leg }} \\
\hline & & & & & & & & \\
\hline & & & white & black & white & black & white & black \\
\hline \multirow[t]{2}{*}{ Equus quagga boehmi } & - & $3.42 \pm 0.65$ & - & - & $1.12 \pm 0.23$ & $1.52 \pm 0.19$ & $2.02 \pm 0.50$ & $1.70 \pm 0.80$ \\
\hline & M & $4.76 \pm 2.06$ & . & & $0.86 \pm 0.53$ & $1.86 \pm 0.56$ & $1.27 \pm 0.64$ & $0.87 \pm 0.33$ \\
\hline \multirow{3}{*}{ Equus grevyi } & M & $2.88 \pm 0.54$ & $0.44 \pm 0.09$ & $0.82 \pm 0.18$ & $0.75 \pm 0.75$ & $1.09 \pm 0.38$ & $0.93 \pm 0.50$ & $1.47 \pm 0.75$ \\
\hline & M & $1.95 \pm 0.73$ & $0.67 \pm 0.14$ & $0.71 \pm 0.27$ & $0.92 \pm 0.33$ & $1.28 \pm 0.49$ & $1.01 \pm 0.48$ & $1.38 \pm 0.52$ \\
\hline & $\mathrm{F}$ & $2.33 \pm 0.66$ & $0.77 \pm 0.11$ & $1.01 \pm 0.34$ & $0.81 \pm 0.27$ & $1.20 \pm 0.35$ & $0.89 \pm 0.36$ & $1.16 \pm 0.45$ \\
\hline \multirow[t]{2}{*}{ Equus burchellii } & $\mathrm{F}$ & $4.37 \pm 0.89$ & - & - & $1.40 \pm 0.70$ & $1.35 \pm 0.59$ & $1.17 \pm 0.81$ & $1.08 \pm 0.61$ \\
\hline & - & $4.77 \pm 0.96$ & - & - & $1.35 \pm 0.76$ & $1.93 \pm 0.42$ & $3.65 \pm 0.68$ & $2.80 \pm 1.18$ \\
\hline Equus quagga burchellii & $\mathrm{M}$ & $2.63 \pm 0.80$ & - & - & - & - & - & - \\
\hline Equus zebra hartmannae & - & $4.90 \pm 0.87$ & $1.79 \pm 0.34$ & $1.39 \pm 0.48$ & $2.67 \pm 0.32$ & $2.20 \pm 0.52$ & $2.64 \pm 0.99$ & $2.37 \pm 0.60$ \\
\hline Tragelaphus eurycerus isaaci & M & - & $1.21 \pm 0.19$ & $9.54 \pm 0.16$ & - & - & - & - \\
\hline \multirow{3}{*}{ Tragelaphus strepsiceros } & M & - & $0.87 \pm 0.13$ & $5.64 \pm 0.85$ & - & - & - & - \\
\hline & $\mathrm{M}$ & - & - & - & - & - & - & - \\
\hline & $\mathrm{M}$ & - & $0.62 \pm 0.39$ & $6.99 \pm 0.31$ & - & - & - & - \\
\hline \multirow{4}{*}{ Tragelaphus imberbis } & M & - & $0.83 \pm 0.22$ & $2.86 \pm 1.26$ & - & - & - & - \\
\hline & M & - & $0.98 \pm 0.17$ & $4.58 \pm 1.77$ & - & - & - & - \\
\hline & M & - & $0.95 \pm 0.10$ & $5.15 \pm 0.51$ & - & - & - & - \\
\hline & $\mathrm{F}$ & - & $0.83 \pm 0.17$ & $3.38 \pm 1.59$ & - & - & - & - \\
\hline \multirow[t]{2}{*}{ Tragelaphus scriptus } & M & - & - & - & - & - & - & - \\
\hline & $F^{*}$ & - & $0.67 \pm 0.07$ & $1.45 \pm 0.17$ & - & - & - & - \\
\hline Okapia johnstoni & $\mathrm{M}$ & $1.84 \pm 0.67$ & - & - & - & - & $2.27 \pm 1.36$ & $3.07 \pm 2.94$ \\
\hline \multirow[t]{2}{*}{ Hyaena hyaena } & M & - & - & - & $0.81 \pm 0.31$ & $1.24 \pm 0.67$ & $0.87 \pm 0.32$ & $1.19 \pm 0.50$ \\
\hline & - & - & - & - & $1.43 \pm 0.40$ & $1.50 \pm 0.53$ & $1.09 \pm 0.39$ & $1.10 \pm 0.19$ \\
\hline \multirow{3}{*}{ Proteles cristatus } & - & - & - & - & $1.31 \pm 1.18$ & $0.58 \pm 0.25$ & $1.21 \pm 0.27$ & $0.75 \pm 0.23$ \\
\hline & - & $1.15 \pm 0.12$ & - & - & $1.20 \pm 0.22$ & $0.93 \pm 0.40$ & $1.64 \pm 0.51$ & $0.61 \pm 0.13$ \\
\hline & - & - & - & - & $1.24 \pm 0.76$ & $1.22 \pm 0.69$ & $1.36 \pm 0.86$ & $1.10 \pm 0.32$ \\
\hline \multirow{3}{*}{ Civettictis civetta } & - & - & - & - & - & - & $1.89 \pm 0.53$ & $2.03 \pm 0.39$ \\
\hline & - & - & - & - & - & - & $1.13 \pm 0.20$ & $1.61 \pm 0.42$ \\
\hline & $\mathrm{F}$ & - & - & - & - & - & $1.46 \pm 0.23$ & $1.96 \pm 0.48$ \\
\hline
\end{tabular}




\section{Ml Macrothink}

\begin{tabular}{|l|c|c|c|c|c|c|c|c|}
\hline & - & - & $2.39 \pm 1.12$ & $2.10 \pm 0.60$ & - & - & - & - \\
& $\mathrm{M}$ & - & $1.66 \pm 0.08$ & $1.61 \pm 0.28$ & - & - & - & - \\
\hline Ictonyx striatus & - & - & $0.80 \pm 0.04$ & $1.18 \pm 0.21$ & - & - & - & - \\
\hline Mungos mungo & $\mathrm{M}$ & - & $0.57 \pm 0.09$ & $0.61 \pm 0.11$ & - & - & - & - \\
& $\mathrm{M}$ & - & $0.85 \pm 0.20$ & $0.84 \pm 0.12$ & - & - & - & - \\
\hline Galidictis fasciata & $\mathrm{F}$ & $1.48 \pm 0.08$ & - & - & - & - & - & - \\
\hline Poecilogale albinucha & $\mathrm{M}$ & - & $1.14 \pm 0.09$ & $0.91 \pm 0.06$ & - & - & - & - \\
\hline
\end{tabular}

Specimens with known gender are marked $\left(\mathrm{M}=\right.$ male, $\mathrm{F}=$ female, $\mathrm{F}^{*}=$ female calf $)$ and unknown gender with (-)

\subsection{Tabanids Attraction Experiments}

There was some variation in the number of tabanids captured in all types of weather during the experimental period. When testing whether the number of captured tabanid flies depended on the type of weather, ANOVA variance analysis revealed significant differences in the number of tabanid flies captured during the entire period $\left(\mathrm{F}_{3,35}=12.642, \mathrm{P}<0.001\right)$.

Tukey HSD $(\mathrm{P}<0.001)$ tests showed differences in the total number of tabanids captured on sunny vs. rainy days, partly cloudy vs. rainy days and partly cloudy vs. cloudy days $(\mathrm{P}=0.048)$ (Figure 3). The highest numbers of tabanids were captured in partly cloudy weather immediately before rainy days, suggesting an association between flight intensity and anticipated rain.

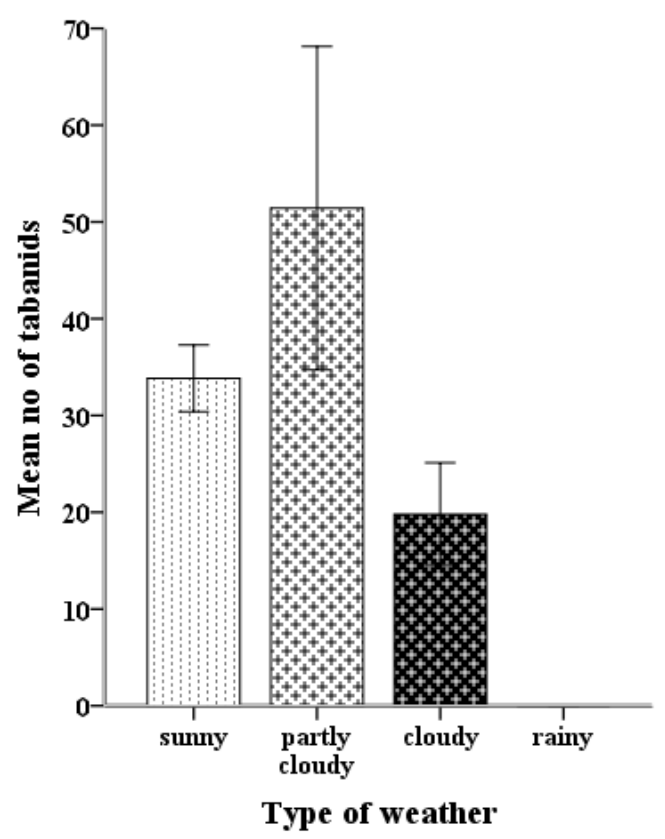

Figure 3. Tabanid flies capture rate depends on weather. Distribution of the total number of tabanids captured in different types of weather during the experimental period.

The number of captured tabanids differed significantly on the sunny (S-pointing) and shady (N-pointing) sides of the glossy sticky panels ( $t$ test: $t=4.132, d f=76, P<0.001$ ). This was also the case for the glossy sticky models ( $t$ test: $t=2.170, d f=76, P=0.033$ ) (Figure 4a, b). 
(a)

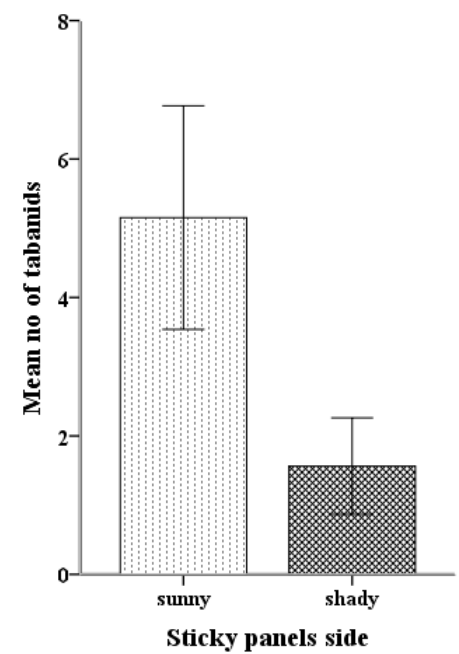

(b)

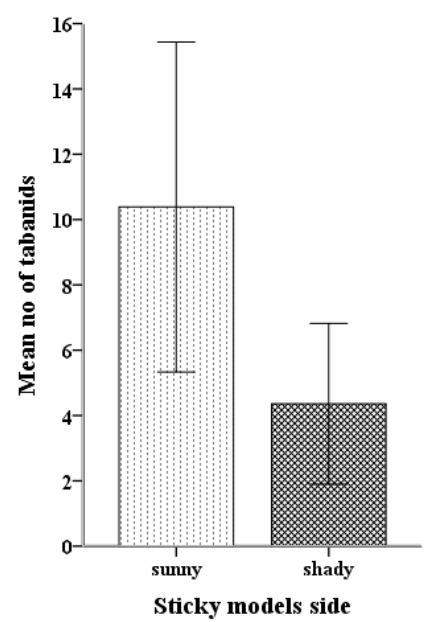

Figure 4. The attractiveness of the sunny and shady sides to tabanids. Comparison of mean tabanids number ( $95 \%$ confidence interval) captured on the two sides of (a) the sticky panels and (b) the sticky models (sunny/shady).

When comparing the difference in number of captured tabanids between the control surfaces (black and white) and, the zebra and the tragelaphidae vertical (Figure 5a) and oblique (Figure $5 b$ ) striped panels, the results revealed significant differences (vertical: ANOVA: $F_{4}$, $190=6.020, P<0.001$; oblique: ANOVA: $\left.F_{4,190}=3.298, P=0.012\right)$. Tukey HSD test $(P<$ $0.001)$ also revealed a significant difference in the number of tabanids between the black and white control panels and the zebra vertical striped panel. Likewise, a significant result was obtained $(P=0.012)$ for differences between the black control panel and both tragelaphidae panels (Figure 5a). Furthermore, Tukey HSD test showed a significant difference $(P=0.01)$ between the black and white control panels and, the zebra oblique striped panel $(\mathrm{P}=0.039$; Figure 5b).

(a)

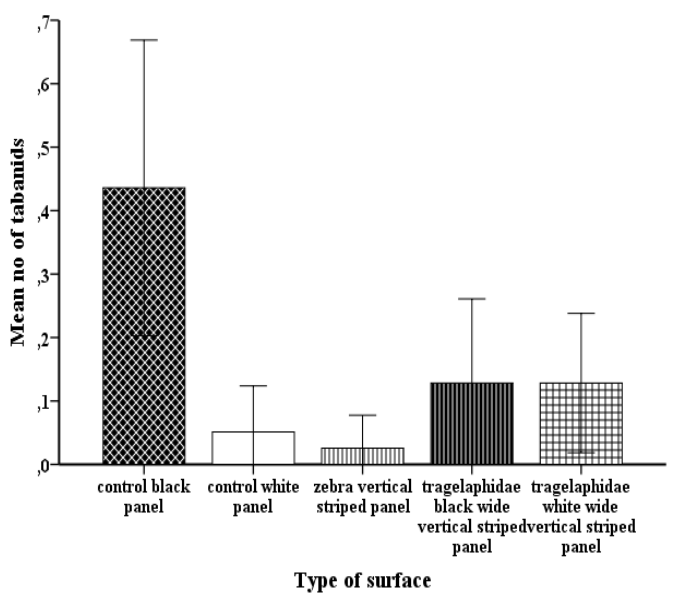

(b)

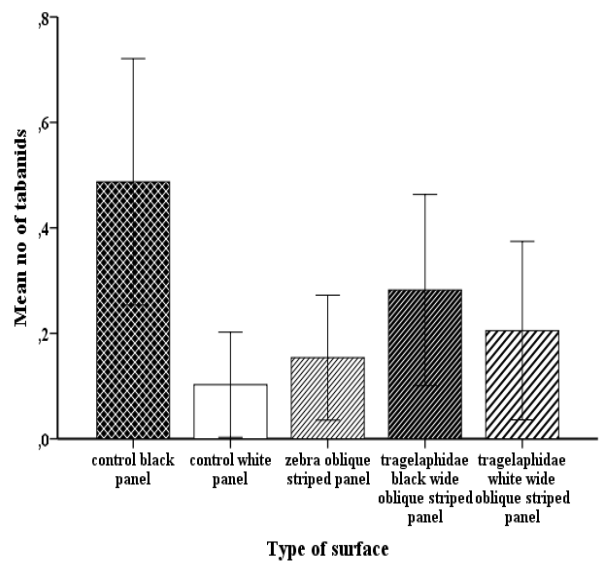

Figure 5. The relationship between control surfaces and, zebra and tragelaphidae vertical (a) and oblique (b) striped panels in terms of attractiveness to tabanids. The surface area for the zebra and tragelaphidae panels is the same as the black and white control areas. 
In terms of tabanids attractiveness there was a highly significant difference between the control panels and the panels with horizontal stripes orientation (ANOVA: $F_{4,190}=12.239, P$ $<0.001)$. According to Tukey HSD test the number of tabanid flies was statistically higher on the control black panel compared to all horizontal striped panels $(P<0.001)$ and respectively, between control black and white panel $(P=0.023)$. The same significant difference was revealed between the control white panel and zebra horizontal striped panel as well as the tragelaphidae white wide horizontal striped panel $(P=0.032$; Figure 6a). With respect to the attractiveness of tabanid flies to the panels placed horizontally on the ground, there was no significant difference in the mean number of tabanids between all the panels $(P=0.883$; Figure 6b).

(a)

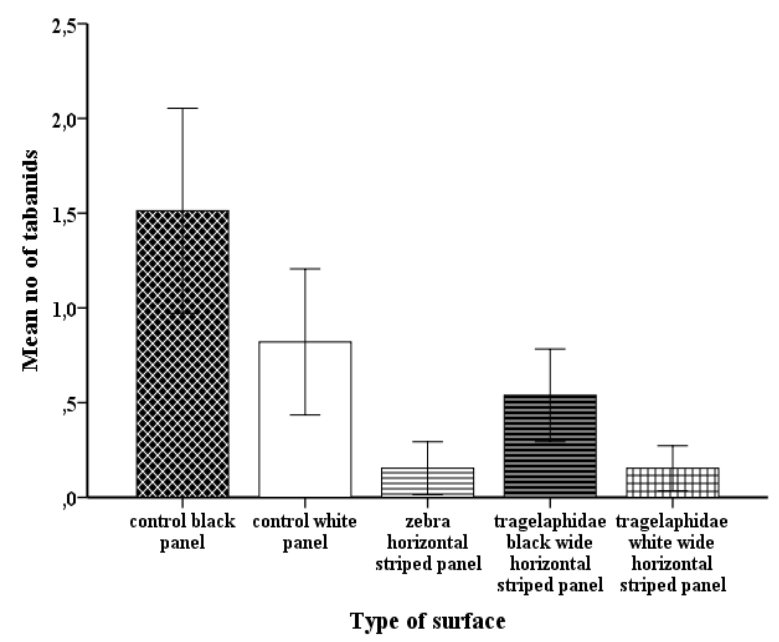

(b)

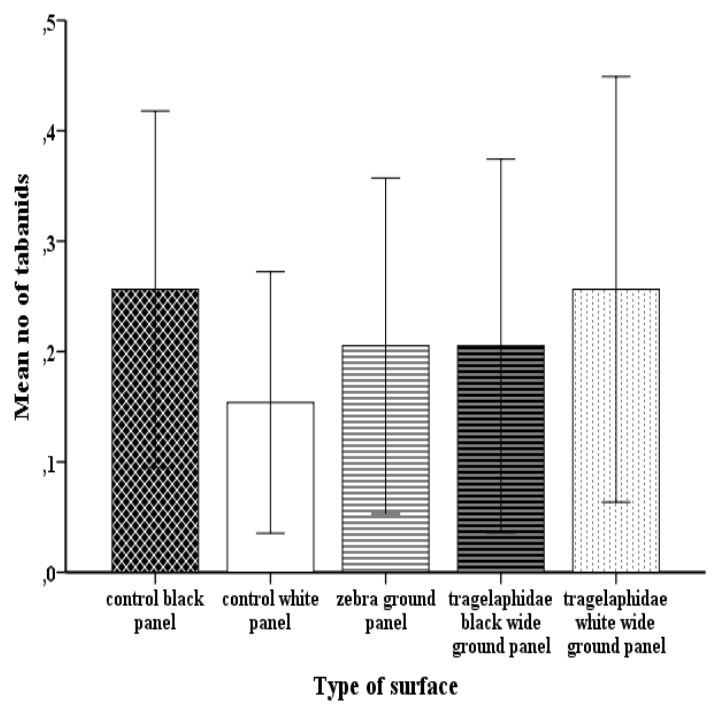

Figure 6. Relationship between control surfaces and, zebra and tragelaphidae horizontal striped panels (a) and striped panels placed on the ground (b) in terms of attractiveness to tabanids. The surface area for the zebra and tragelaphidae panels is the same as the black and white control areas.

With regards to number of tabanid flies attracted to control surfaces and zebra models with different stripes orientation (vertical, horizontal and oblique), ANOVA revealed a significant difference $\left(F_{4,190}=5.562, P<0.001\right)$. Tukey HSD test showed differences in number of tabanids attracted to the control black model and the other types of surfaces (control black model and zebra vertical striped area $P=0.005$; control black model and zebra horizontal striped area $P=0.002$ and respectively, control black model and zebra oblique striped area $P=0.005$; Figure 7a). Likewise, the number of tabanid flies attracted to control surfaces models and tragelaphidae model was significantly different (ANOVA: $F_{3,152}=5.040, P=$ 0.002). Tukey HSD test revealed differences in the mean number of tabanids only between the control black model and both types of tragelaphidae striped areas (control black model and tragelaphidae abdomen vertical striped area $P=0.016$ and respectively, control black model and tragelaphidae rump vertical striped area $P=0.004$; Figure $7 \mathrm{~b}$ ). 
(a)

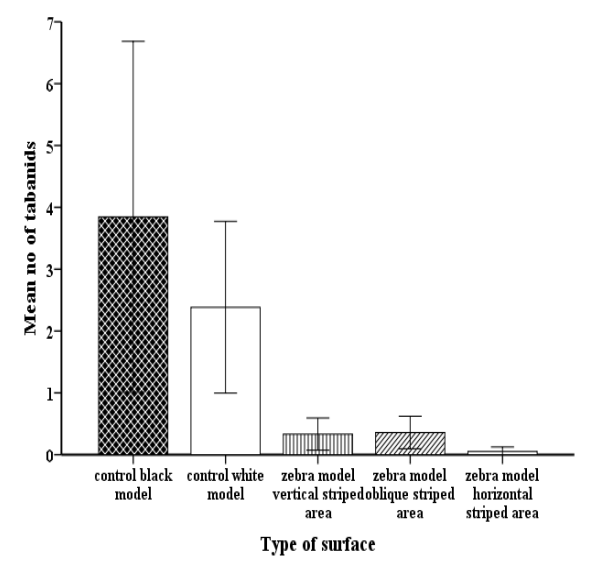

(b)

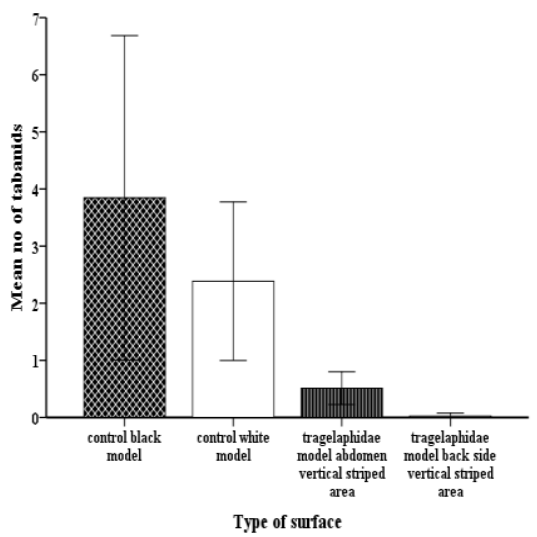

Figure 7. The relationship between (a) control surfaces and the zebra model (vertical, oblique and horizontal striped areas: (a)) and the tragelaphidae model (abdomen and rump vertical striped areas: (b)). Comparison of control surfaces (black and white) and zebra model in mean number of captured tabanids (a). Furthermore, the second comparison has been made between control surfaces and tragelaphidae model in mean number of tabanids caught on these surfaces (b).

The difference in number of captured tabanids on control surfaces models and okapi model (black and horizontal striped areas) was significant (ANOVA: $F_{3,152}=3.181, P=0.026$ ). Tukey HSD test, $(P=0.014)$ pointed out a significant difference between the control black model and the okapi model horizontal striped area (Figure 8a). Furthermore, ANOVA revealed a significant difference $\left(F_{3,152}=4.252, P=0.006\right)$ between the numbers of tabanid flies caught on control surfaces models and the hyaenidae model. The Tukey HSD test also showed significant differences $(P=0.031)$ between the control black model and the hyaenidae model abdomen vertical striped area and also $(P=0.009)$ between the control black model and the hyaenidae model leg horizontal striped area (Figure 8b).

(a)

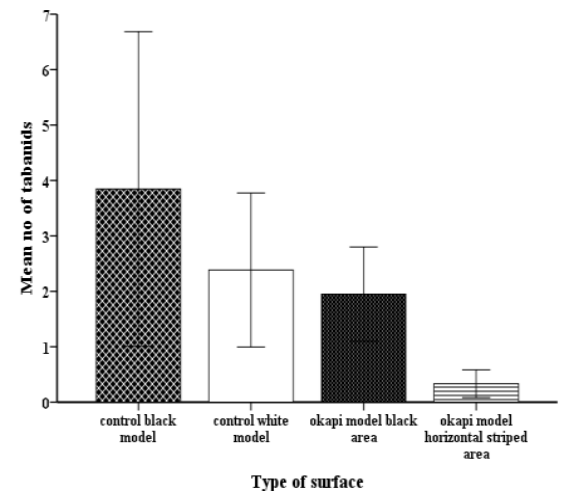

(b)

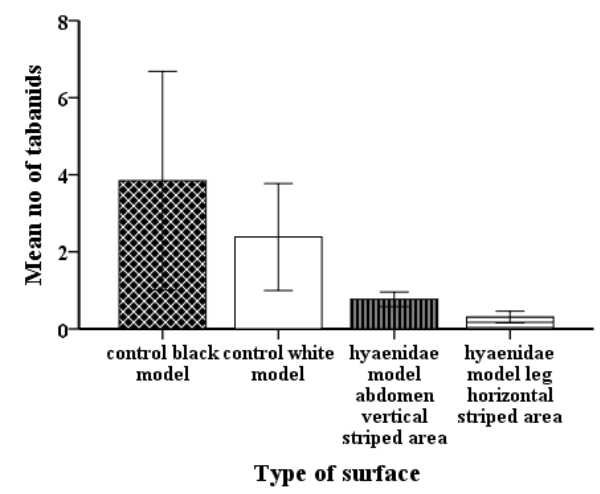

Figure 8. Relationship between control surfaces and okapi model (black and horizontal striped area: (a)) and the hyaenidae model (abdomen vertical and leg horizontal striped areas: (b)). Comparison of control surfaces (black and white) and okapi model in mean number of tabanids caught on the entire period (a). Likewise, the second comparison has been made between control surfaces and hyaenidae model (b). 


\section{Discussion}

\subsection{Measurements of Stripe Width in Zebra and Some African Mammal Coats}

I found in all species of zebra very similar characteristics of coat patterns, three types of stripes orientation (vertically, horizontally and oblique) but variable widths of stripes in each individual. The patterning of these mammalian coats is probably due to mechanisms based on reaction-diffusion kinetics invented by Turing (1952) and the stripes in zebra were shown to have arisen owing to inhibition not stimulation of melanin (Murray, 1981). The zebras were originally dark animals with white stripes where the pigmentation has been inhibited (Prothero and Schoch, 2003).

For Equidae the decision to generate pigmentation occurs approximately at three, four or five weeks of development depending on the species of zebra (Bard, 1981). Natural selection can likely explain the appearance and development of the stripes that have evolved on many mammalian coats. Since striped species occur in many families and in all orders of mammals, the stripes have probably evolved many times during evolution. Several reasons have been offered for the development of a striped coat pattern, all of which have been discussed in two recent reviews by Ruxton (2002) and Caro (2009) and which is also discussed in Kingdon $(1977,1979,1982)$.

Some families have many striped species compared to other families, and in some species it is only the young that carry a striped coat pattern (i.e. it is absent in the adults). The female calf in Tragelaphus scriptus has stripes on its back and abdomen whereas the adult male only has stripes on the abdomen (Kingdon 1979, 1982; Nowak 1999). Equus quagga burchellii, from an old specimen from 1907, has no stripes on its legs and the stripes on the rump are more attenuated compared to the other zebra species. This specimen is very similar to individuals from an extremely small population from Zululand and western Kalahari that has a very similar coat pattern (including white legs). Looking at the rarity of this pattern means that it was probably exposed to a strong selection which has favored the current repetitive stripes patterns (Kingdon, 1979).

In the museums the measurement characteristics (stripe widths of black and white color) were taken on thirty-six African mammals coats. All displayed different striped and spotty patterns. In my work (in terms of statistical analyses) I was particularly interested in the species that live in more open habitats. I also included in the experiment and analysis two species of the family Hyaenidae to see how attractive to tabanid flies the stripes of carnivorous species are, compared to those of grazing species.

The stripe widths for the models were obtained by averaging the values from all individuals of all species within the same family. Thus, any individual variation was removed and a general model of stripes was obtained. This is representative not only for all the individuals within the same species, but also for all species within the same family.

I found similarities in numbers of stripes and means of stripe widths between sexes (of Equus grevyi and Tragelaphus imberbis) but I was not able to perform any statistical test because I had too few individuals for each sex. In Equus grevyi the behavior and social system are 
related to the spatial and temporal distribution of their resources. In terms of sexual activity there is a peak during rainfall periods. This species of zebra is the most territorial species with established territories only during the rainy season that include the territorial males, mares and their foals. For half of the year the individuals are spread out across the dried landscape. In this polygamous species mate choice does not depend on the presence of stripes and the females that exist in estrus, thus provide a powerful stimulus and, are awarded to the territorial stallion in this territorial system. In a non-territorial system there is much expenditure of energy and time by non-territorial males and strong competition (Kingdon, 1979).

One may expect a strong attack rate of tabanid flies prior to rain and in the early part of the rainy season. So as Equus grevyi is occupied with mating and territorial defense, and has little time to rest in a shaded area, the attack rate and disease transmission may be expected to be high during this part of the year. Hence, a striped coat pattern might act as an efficient protection against tabanid attacks for the zebras during this important stage of their life. In Tragelaphus imberbis there is sexual dimorphism, with the male being heavier than the female and with traits (such as horns) not found in females. The stripes and other markings, such as white patches on neck. are present in both sexes. The stripes on the body seem not to be involved in sexual selection, but they can display a visual impression that is extremely distinctive and probably could help in communication (Kingdon, 1982). However, according to my results the striped pattern may also decrease the number of blood-sucking tabanids flies that they attract, suggesting a potential involvement in protection against parasite attacks.

\subsection{Tabanids Attraction Experiments}

The main finding of the tabanids attraction experiments was that the characteristics of striped patterns were obviously repellent to tabanid flies. I caught a total of 864 tabanid flies belonging to five species that were attracted to the experimental surfaces (Mandahl-Barth and Coulianos, 1985). I also found a smaller number of captured tabanids on panels placed vertically compared with the number of tabanid flies found on my models. This, may be explained by the fact that the tabanids were less attracted to vertical surfaces because they do not reflect non-horizontally polarized light. My models were arranged with 30 degree sloping sides, causing some linearly polarized light to be reflected at an angle relative to the sky and perhaps leading to a more attractive signal for tabanid flies. Furthermore, the top of the models was located higher than the top edge of the panels, and flies flying higher above the ground might be more easily attracted and captured.

Tabanids frequently landed on my models, especially on the upper side of both sunny and shady sides. Those regions probably reflected horizontally polarized light that stimulates the ventral eye region of tabanids, an eye region that sub serves positively polarotactic behavior in these flies (Horváth, et al., 2008), resulting in attraction and landing on the surface.

Many tabanid flies were attracted to the sunny sides of the two types of experimental surfaces (in warm weather). One reason might be that the tabanids need a higher body temperature for flight and when trying to escape the animal's reactions during their attacks. They showed preferences to fly more in partly cloudy and sunny weather compared to rainy days. I also 
found that the effect of rain played a major role in attracting tabanids because on most days before rain, the number of captured tabanid flies increased during the collection period. This suggests higher flight activity prior to good egg laying conditions, when the soil and mud will be wet by the rain. In particular, due to their need to keep their body surface hydrated, Haematopota pluvialis, a tabanid species that was captured to a greater extent, prefers to fly under approaching thunderstorms or rain periods (Mandahl-Barth and Coulianos, 1985). There is a possibility that tabanid flies looking for egg-laying places close to water may be active under different types of weather, both under sunny and overcast conditions. However, while searching for a host to feed on they may be more active under sunny or partly cloudy skies (Horváth, et al., 2010), as was also observed in this study.

The behavior of the captured tabanid flies was not surprising because they were more attracted by homogeneous colors (black or white) compared with all striped patterns. I observed that each striped animal model was extremely repellent to tabanids while homogeneously black or even white color was more attractive to tabanid flies. The black body surface on the Okapi was particularly attractive to all five species of tabanid flies. The black color has been shown to be especially attractive to tabanids due to the polarizing properties of a dark coat (Horváth, et al., 2010), and it was this part of the okapi model that attracted the most tabanids in my experiments. I found that when stripes were used the number of tabanids was reduced, and the reason seems to be the increased repellency by the striped pattern. This observation suggests an evolutionary benefit for African mammals from the introduction of white stripes onto a black/brown coat, avoidance of attacks by blood-sucking tabanids.

Severe consequences from tabanid attacks have been observed for grazing animals, for which intense tabanid flies attack rates can reduce the foraging time as well as milk production (Lehane, 2005). The hosts will also suffer from an increased risk of disease transmission if they have to withstand intense attack rates of biting flies (Foil, 1989). In Africa, large antelopes (greater kudu and eland) inhabiting the Tanganyika region have been found to suffer trypanosome infections as result of tabanids such as Tabanus taeniola, $T$. ditaeniatus, $T$. par, T. biguttatus, T. africanus and Haematopota species. In central and west Africa some species of Chrysops may transmit parasites such as Loa loa to humans, causing the infection of eyes (Vanderplank, 1944; Lehane, 2005). For all these reasons, host mammals would benefit by avoiding attacks as much as possible. There might be at least two ways by which attacks could be avoided. First, the mammal may inhibit the reflected horizontally polarized light signal used by the tabanids, by introducing spotty or striped coat patterns. Second, they may avoid being sun-exposed at the time of the day and during weather conditions when the risk of being attacked is largest. However, the escape to shade will result in a decreased time for grazing, which might have negative fitness consequences for grazing mammals. Furthermore, mammals inhabiting forested and bushy areas are the only ones able to easily find a shaded shelter. African mammals inhabiting the open plains like the zebras, giraffe and kudu might have less chance to escape from the sun and tabanid attacks. Therefore, in those grazing mammal species the introduction of a striped or spotty coat pattern seems to be an excellent mechanism for reducing tabanids attacks. 
The Hyaenidae show wider and lighter stripes and on the areas where stripes were present, the attractiveness to tabanids was lower compared with the control black model. However, for Hyaenidae, (Hyaena hyaena species), which are strictly nocturnal (the result of Crocuta crocuta persecution), there are presumably other factors involved in the evolution of the stripes on their coats. This species often needs to hide when hunting, and thus, the coat pattern may be used for camouflage. My model experiment, however, suggested that the tabanids attack rate was also reduced in a model imitating the striped coat pattern of Hyaenidae. The same situation may be found in Proteles cristatus which is both diurnal and nocturnal. Thus, in both species the appearance of stripes might be involved in social interactions, as camouflage (Kingdon, 1977), or may also act as protection against tabanids. Small stripe widths (on the head and legs) are not attractive to tabanid flies compared with wider stripes on the neck, back and rump. However, the head and legs have a thin skin that ought to be protected against tabanid attacks. Several of the carnivorous mammals I measured in my study are active at dusk and dawn, or at night with little activity around midday, when tabanids are most active. Some of them even take shelter or sleep in underground nests, termitaries or rock crevices (Kingdon, 1977). It is less likely that they are exposed to the attacks of tabanid flies to the same degree as the grazing mammals of the African open plains (zebra, greater and lesser kudu, okapi, giraffe and so on). Thus, in these carnivorous mammals there are probably other reasons for the evolution of striped and spotty coat patterns (Caro, 2009). Overall my work suggests that there seems to be a general reduction in attractiveness with the introduction of stripes that culminates in least attraction for even and narrow stripes (like those in the zebra). However, as long as some stripes are introduced, the animal may benefit from not being attacked by tabanids flies quite as frequently, as the reflected polarization signal is broken into smaller areas. Avoidance of tabanids may thus have been a selective force during the evolution of striped coat patterns in grazing mammals, as hypothesis that is supported by my work. Of course, tabanids may also use other sources of information (like odors) to find their hosts, and further investigations on the relative effects of striped coat patterns and host odors are needed to understand the complete picture of this host-parasite system.

\section{Acknowledgments}

I am deeply grateful to Sven-Axel Bengtsson and Lars Lundberg at the Zoological Museum in Lund, Ulf Johansson and Peter Nilsson at the Swedish Museum of Natural History in Stockholm, Mogens Andersen at the Natural History Museum of Denmark in Copenhagen and Ionut Tausan at the Hunting Museum "August von Spiess" Sibiu, Romania for supplying me with all the available coats and stuffed animals for measurements. I acknowledge Per Ola Olsson from Vankiva, Hässleholm, Sweden for his agreement to run the field experiments on his property and Mikael Sörensson for invaluable help with the identification of some of the samples. I would like to thank my husband Michael for helping me to build all the materials and their installation in the field.

I acknowledge Susanne Åkesson, Lund University for supervising the project (Master Thesis). This study was financed by a research grant from the Swedish Research Council to Professor Susanne Åkesson. 


\section{Macrothink}

Journal of Biology and Life Science ISSN 2157-6076 2016, Vol. 7, No. 1

I am deeply grateful to the readers of the manuscript, Professors Eric Warrant and Thomas Pape, Mogens Andersen and other anonymous readers for their extremely valuable feedback.

\section{References}

Bard J. B. L. (1981). A model for generating aspects of Zebra and other mammalian coat patterns. Journal of Theoretical Biology, 93, 363-385. http://dx.doi.org/10.1016/0022-5193 (81)90109-0

Caro T. (2009). Contrasting coloration in terrestrial mammals. Philosophical Transaction of the Royal Society B, 364, 537-548. http://dx.doi.org/10.1098/rstb.2008.0221

Chinery M. (2007). Insects of Britain and Western Europe. London: A \& C Publishers Ltd

Chvála M., Lyneborg L., Moucha J. (1972). The horse flies of Europe (Diptera, Tabanidae). Copenhagen: Entomological Society of Copenhagen

Foil L. D. (1989). Tabanids as vectors of disease agents. Parasitology Today, 5, 88-96. http://dx.doi.org/10.1016/0169-4758 (89)90009-4

Godfrey D., Lythgoe J. N., \& Rumball D. A. (1987). Zebra stripes and tiger stripes: the spatial frequency distribution of the pattern compared to that of the background is significant in display and crypsis. Biological Journal of the Linnean Society, 32, 427-433. http://dx.doi.org/10.1111/j.1095-8312.1987.tb00442.x

Horváth G., Majer J., Horváth L., Szivák I., \& Kriska G. (2008). Ventral polarization vision in tabanids: horseflies and deerflies (Diptera: Tabanidae) are attracted to horizontally polarized light. Naturwissenschaften, 95, 1093-1100. http://dx.doi.org/10.1007/s00114-008-0425-5

Horváth G., Blahó M., Kriska G., Hegedüs R., Gerics B., Farkas R., \& Åkesson S. (2010). An unexpected advantage of whiteness in horses: the most horsefly-proof horse has a depolarizing white coat. Proceeding of the Royal Society B, 00. 1-8. http://dx.doi.org/10.1098/rspb.2009.2202

Kingdon J. (1977). East African Mammals. An Atlas of Evolution in Africa. vol IIIA, Carnivores. University of Chicago Press edition 1989. London: Academic Press (Inc.) London Ltd

Kingdon J. (1979). East African Mammals. An Atlas of Evolution in Africa. vol IIIB, Large Mammals. London: University of Chicago Press edition 1989. London: Academic Press (Inc.) London Ltd

Kingdon J. (1982). East African Mammals. An Atlas of Evolution in Africa. vol IIIC, Bovids. London: University of Chicago Press edition 1989. London: Academic Press (Inc.) London Ltd

Lehane M. J. (2005). The Biology of Blood-Sucking in Insects $\left(2^{\text {nd }}\right.$ edition). Cambridge: Cambridge University Press. http://dx.doi.org/10.1017/CBO9780511610493

Mandahl-Barth G., Coulianos C. C. (1985). Vad jag finner i skogen. Stockholm: P.A. Norstedt 
\& Söners Förlag

Morris D. (1991). Animal watching: A field guide to animal behaviour. London: Arrow Books Limited

Murray J. D. (1981). A pre-pattern formation mechanism for animal coat markings. Journal of Theoretical Biology, 88, 161-199. http://dx.doi.org/10.1016/0022-5193(81)90334-9

Nowak R. M. (1999). Walker's mammals of the world. vol 1. Baltimore: John Hopkins University Press

Ortolani A. (1999). Spots, stripes, tail tips and dark eyes: Predicting the function of carnivore colour patterns using the comparative method. Biological Journal of the Linnean Society, 67, 433-476. http://dx.doi.org/10.1111/j.1095-8312.1999.tb01942.x

Prothero D. R., \& Schoch R. M. (2003). Horns, Tusks, and Flippers: The Evolution of Hoofed Mammals. Baltimore: John Hopkins University Press

Ruxton G. D. (2002). The possible fitness benefits of striped coat coloration for zebra. Mammal Review, 32, 237-244. http://dx.doi.org/10.1046/j.1365-2907.2002.00108.x

Vanderplank F. L. (1944). Apparent densities of certain African blood-sucking insects (Diptera). Proceeding of the Royal Entomological Society of London, 19, 68-72. http://dx.doi.org/10.1111/j.1365-3032.1944.tb01115.x

\section{Copyright Disclaimer}

Copyright for this article is retained by the author(s), with first publication rights granted to the journal.

This is an open-access article distributed under the terms and conditions of the Creative Commons Attribution license (http://creativecommons.org/licenses/by/3.0/). 